ESAIM: PROCEEDINGS AND SURVEYS, January 2015, Vol. 48, p. 400-419

N. Champagnat, T. Lelièvre, A. Nouy, Editors

\title{
DIFFUSION LIMIT OF THE SIMPLIFIED LANGEVIN PDF MODEL IN WEAKLY INHOMOGENEOUS TURBULENCE
}

\author{
Casimir Emako ${ }^{1}$, Viviana Letizia ${ }^{2}$, Nadezda Petrova ${ }^{3}$, Rémi Sainct $^{4}$, Roland $^{2}$ \\ DUClOUS ${ }^{5}$ AND Olivier SOUlARD ${ }^{5}$
}

\begin{abstract}
In this work, we discuss the modelling of transport in Langevin probability density function (PDF) models used to predict turbulent flows [1]. Our focus is on the diffusion limit of these models, i.e. when advection and dissipation are the only active physical processes. In this limit, we show that Langevin PDF models allow for an asymptotic expansion in terms of the ratio of the integral length to the mean gradient length. The main contribution of this expansion yields an evolution of the turbulent kinetic energy equivalent to that given by a $k-\varepsilon$ model. In particular, the transport of kinetic energy is given by a gradient diffusion term. Interestingly, the identification between PDF and $\bar{k}-\bar{\varepsilon}$ models raises a number of questions concerning the way turbulent transport is closed in PDF models. In order to validate the asymptotic solution, several numerical simulations are performed, with a Monte Carlo solver and also with a deterministic code.

Résumé. Dans cet article, nous abordons la question de la modélisation du transport turbulent dans les modèles de turbulence basés sur les fonctions de densité de probabilité (PDF). Nous étudions la limite diffusive de ces modèles obtenue lorsque l'advection et la dissipation sont les seuls processus physiques actifs. Dans cette limite, nous montrons que les modèles PDF donnent lieu à un développement asymptotique selon un petit paramètre correspondant au rapport de l'échelle intégrale sur l'échelle du gradient moyen. La contribution principale de ce développement s'identifie avec un modèle $\bar{k}-\bar{\varepsilon}$ classique. En particulier, le transport de l'énergie turbulente est donné par une diffusion en premier gradient. L'identification entre modèle $\bar{k}-\bar{\varepsilon}$ et modèle PDF permet de soulever un certain nombre de questions sur la manière dont le transport est modélisé dans les approches PDF. La solution asymptotique est validée par des simulations numériques réalisées à l'aide d'un code Monte Carlo mais aussi d'un code déterministe.
\end{abstract}

\section{InTRODUCTION}

Since the early work of Pope [1], the so-called probability density function (PDF) approach has proved to be an efficient tool for predicting turbulent flows. In this approach, one derives and solves a modelled transport equation for the one-point PDF of the fluctuating velocity field and, when necessary, of additional variables describing the state of the flow, such as concentration, temperature or density. In the modelling process of the flow one-point statistics, closures must be applied to the turbulent acceleration as well as to molecular diffusion

${ }^{1}$ UPMC Univ Paris 06, UMR 7598, Laboratoire Jacques-Louis Lions, F-75005, Paris, France

${ }^{2}$ Univ Paris-Dauphine, UMR 7534, Laboratoire Ceremade, F-75016, Paris, France

3 ONERA, DEFA, F-91123 Palaiseau, France

${ }^{4}$ Ecole des Ponts ParisTech, Laboratoire Cermics

${ }^{5}$ CEA, DAM, DIF, F-91297, Arpajon, France

(C) EDP Sciences, SMAI 2015 
terms. Most of these closures yield a PDF transport equation of the Langevin type [1-4]. In this work, we will only focus on this class of models.

While mostly used to predict turbulent reactive flows, the PDF approach has also demonstrated its utility for solving incompressible inert flows. In this context, Langevin PDF models have been shown [2] to be connected to simpler turbulent models which focus solely on the second-order one-point correlation tensor of the velocity field, also called Reynolds stress tensor. These Reynolds stress models (RSM) revert to the well known $k-\varepsilon$ model when turbulence is isotropic. The PDF/RSM equivalence encompasses most physical processes at work in incompressible flows, including production, non-linear redistribution and dissipation effects. However, strong differences exist in the way both approaches deal with the transport of the turbulent kinetic energy and of its anisotropy.

In RSM, turbulent transport is usually modelled by a gradient diffusion assumption. Many variants of this closure exist, but most are found to yield similar results in practical situations [5,6]. In the PDF approach, the situation is different. The advection term appearing in the Navier-Stokes equations does not require any closure. In that sense, turbulent advection is often said to be treated "exactly" or "without assumption" $[4,7]$. However, such statements might be somewhat misleading. Indeed, the overall process of turbulent transport is not exact since the statistics of the velocity field are affected by the Langevin closures used in the remaining parts of the PDF transport equation.

Thus, turbulent transport and Langevin closures are interacting in PDF models. This interaction is flowdependent and cannot be made explicit in the general case. Yet, when non-equilibrium/production effects become negligible, the PDF equation is expected to degenerate and to yield a gradient diffusion formulation for the transport of Reynolds stresses. This is suggested by several works, for instance $[2,5,8]$, which focus on triple velocity correlations and on their expression in the absence of production. As a consequence, in this diffusion limit, a PDF/RSM equivalence should exist for the turbulent transport term. Then, significant knowledge could be gained by comparing the two families of models, just as it was done in the homogeneous case by Pope [2].

However, the diffusion limit of PDF models has never been looked at thoroughly. The precise conditions under which it occurs have not been made explicit. Besides, the influence of dissipation processes are usually discarded while they are expected to play a significant role. Finally and more importantly, the study of the diffusion limit has been limited to considerations on the sole triple velocity correlations and not on the PDF itself.

Thus, the purpose of this work is to study the diffusion limit of PDF Langevin models and to explicit the connection with RSM models in that particular case. To this end, we consider a simplified setting in which diffusion and dissipation are the only active physical mechanisms. Besides, we restrict our attention to the simplified Langevin model described for instance in [3]. Then, we look for an asymptotic expansion of the Eulerian Langevin PDF equation in terms of the ratio of the integral length to the mean gradient length. The relevance of this expansion is verified on several simulations. Finally, its implications in terms of physical models are discussed.

\section{Simplified LANGevin PDF model APPlied to A TURBUlent ZONE}

Throughout this work, we will consider a canonical turbulent flow consisting in a $1 \mathrm{D}$ slab of turbulence that decays and diffuses with time, and has no mean velocity. This flow is sketched in Figure 1 and will be referred to as turbulent zone (TZ). The inhomogeneous direction is denoted by $x_{1}$ and the length of the TZ by $L_{T Z}$. Our interest lies in finding the properties of the Eulerian PDF $f\left(\mathbf{u} ; x_{1}, t\right)$ of the velocity field $\mathbf{u}=\left(u_{1}, u_{2}, u_{3}\right)$ at point $x_{1}$ and time $t$ when modelled by the simplified Langevin model (SLM) [1]. The PDF $f$ is defined as:

$$
f\left(\mathbf{u} ; x_{1}, t\right)=\overline{\delta(\boldsymbol{v}(\boldsymbol{x}, t)-\mathbf{u})},
$$

where $\boldsymbol{v}(\boldsymbol{x}, t)$ is the instantaneous value of the velocity field, $\delta$ is the Dirac delta function and where, for any quantity $a, \bar{a}$ represents the averaging operator. Note that the PDF $f$ depends on $x_{1}$ instead of $\boldsymbol{x}$ because of 


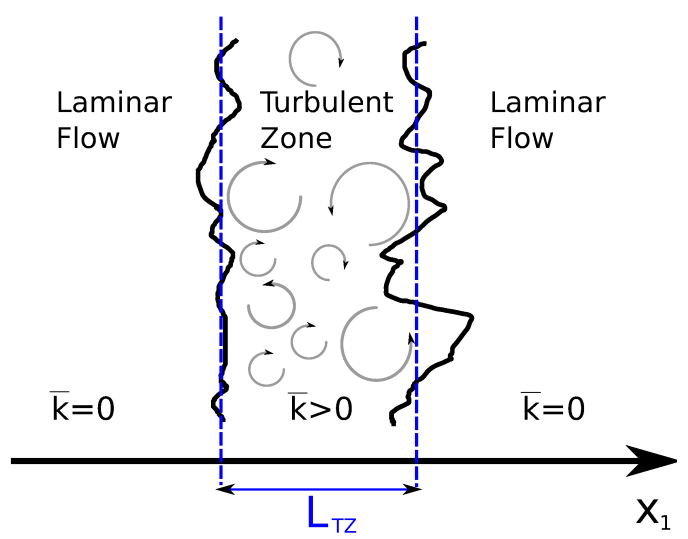

FigURE 1. Sketch of a turbulent zone as studied in this work.

the assumed statistical homogeneity of the flow in the directions $x_{2}$ and $x_{3}$. In the TZ configuration and with the SLM, the evolution of $f$ is given by:

$$
\frac{\partial f}{\partial t}+u_{1} \frac{\partial f}{\partial x_{1}}=-\frac{\partial}{\partial u_{j}}\left[\left(\frac{\partial R_{1 j}}{\partial x_{1}}-\frac{C_{1}}{2} \bar{\omega} u_{j}\right) f\right]+\frac{C_{0}}{2} \bar{\varepsilon} \frac{\partial^{2} f}{\partial u_{j} \partial u_{j}},
$$

where $C_{0}$ and $C_{1}$ are model constants, $R_{i j}=\overline{u_{i} u_{j}}$ is the Reynolds stress tensor, $\bar{k}$ is the mean turbulent kinetic energy, $\bar{\omega}$ is the mean dissipation frequency and $\bar{\varepsilon}$ is the mean dissipation rate. Note the presence of the gradient of the Reynolds stress tensor in the right-hand side of Equation (2.1). This is due to the fact that the mean velocity is null and that the first moment of $f$ is zero: $\int u_{i} f\left(\mathbf{u} ; x_{1}, t\right) d \mathbf{u}=0$ (see eq. (12.19) in [3]). The Reynolds stress tensor $R_{i j}$ and $\bar{k}$ are obtained directly from the PDF by the relations:

$$
\bar{k}\left(x_{1}, t\right)=\frac{1}{2} R_{i i}\left(x_{1}, t\right)=\frac{1}{2} \overline{u_{i} u_{i}}\left(x_{1}, t\right) \quad \text { and } \quad R_{i j}\left(x_{1}, t\right)=\overline{u_{i} u_{j}}\left(x_{1}, t\right)=\int_{\mathbb{R}^{3}} u_{i} u_{j} f\left(\mathbf{u} ; x_{1}, t\right) d \mathbf{u} .
$$

In these expressions, the Einstein summation convention on indices is used. The mean dissipation rate and mean frequency are linked by the relation:

$$
\bar{\omega}\left(x_{1}, t\right)=\frac{\bar{\varepsilon}\left(x_{1}, t\right)}{\bar{k}\left(x_{1}, t\right)}
$$

An additional equation for the dissipation is required to close the system. As in standard $\bar{k}-\bar{\varepsilon}$ models, this equation is obtained by direct analogy with the equation of $\bar{k}$. The evolution of $\bar{k}$ deduced from the PDF equation (2.1) is:

$$
\frac{\partial \bar{k}}{\partial t}+\frac{\partial}{\partial x_{1}}\left(\overline{u_{1} k}\right)=-\bar{\varepsilon}
$$

The evolution of $\bar{\varepsilon}$ is then set to:

$$
\frac{\partial \bar{\varepsilon}}{\partial t}+\frac{\partial}{\partial x_{1}}\left(C_{\varepsilon} \bar{\omega} \overline{u_{1} k}\right)=-C_{\varepsilon_{2}} \bar{\omega} \bar{\varepsilon}
$$

where $C_{\varepsilon}$ and $C_{\varepsilon_{2}}$ are model constants and where $\overline{u_{i} k}$ is the following triple velocity correlation:

$$
\overline{u_{i} k}=\frac{1}{2} \overline{u_{i} u_{p} u_{p}} .
$$


The values of the different constants appearing in the above equations are given in table 1 . These values are taken from the literature [1-4].

\begin{tabular}{cccc}
\hline$C_{0}$ & $C_{1}$ & $C_{\varepsilon}$ & $C_{\varepsilon_{2}}$ \\
$\frac{2}{3}\left(C_{1}-1\right)$ & {$[1.5,5]$} & 1 & 1.9 \\
\hline TABLE 1. & Model constants
\end{tabular}

Note that $C_{0}$ and $C_{1}$ are not independent: in order to ensure that $\bar{\varepsilon}$ is the dissipation rate of $\bar{k}$ one must have $C_{0}=\frac{2}{3}\left(C_{1}-1\right)$. As noted in [2], the value of $C_{1}$ varies significantly in the literature. It mostly depends on whether the SLM is used to model both the non-linear redistribution of energy and the rapid contribution of the pressure gradient, or whether it is associated with an additional component modelling the rapid pressure part. In the former case, the value of $C_{1}$ is usually set to higher values, typically $C_{1}=4.15$. In the latter case, it is set to lower values, typically $C_{1}=1.8$. In the absence of production, as in the TZ case considered here, there is no rapid pressure term and both low and high values of $C_{1}$ are acceptable.

\section{WeAKLY INHOMOGENEOUS LIMIT AND DIFFUSION REGIME}

\subsection{Main assumption}

Two main lengths characterize the turbulent field in the TZ configuration, the integral length $\ell$ and the gradient length $L$. They are respectively defined as:

$$
\ell=\frac{\bar{k}^{3 / 2}}{\bar{\varepsilon}} \quad \text { and } \quad L=\left[\frac{1}{\bar{k}} \frac{\partial \bar{k}}{\partial x_{1}}\right]^{-1} .
$$

The integral length $\ell$ is representative of the size of the turbulent eddies present in the turbulent zone, while $L$ measures the inhomogeneity of the turbulent field. Its maximum is expected to be roughly on the order of the turbulent zone size $L_{T Z}$.

We now make the assumption that the flow is weakly inhomogeneous, i.e. that turbulent eddies are much smaller than $L$. More precisely, we assume that:

$$
\frac{\ell}{L} \leq \epsilon_{a} \ll 1
$$

where $\epsilon_{a}$ is a constant. Anticipating on a configuration where the PDF remains close to a Gaussian, this assumption can be incorporated in Equations (2.1) and (2.5) by introducing a rescaled spatial coordinate:

$$
z=\frac{x_{1}}{\epsilon_{a}}
$$

With this definition, Equations (2.1) and (2.5) can be rewritten as:

$$
\begin{aligned}
& \frac{\partial f}{\partial t}+\epsilon_{a} u_{1} \frac{\partial f}{\partial z}=-\frac{\partial}{\partial u_{j}}\left[\left(\epsilon_{a} \frac{\partial R_{1 j}}{\partial z}-\frac{C_{1}}{2} \bar{\omega} u_{j}\right) f\right]+\frac{C_{0}}{2} \bar{\varepsilon} \frac{\partial^{2} f}{\partial u_{j} \partial u_{j}} \\
& \frac{\partial \bar{\varepsilon}}{\partial t}+\epsilon_{a} \frac{\partial}{\partial z}\left(C_{\varepsilon} \bar{\omega} \overline{u_{1} k}\right)=-C_{\varepsilon_{2}} \bar{\omega} \bar{\varepsilon}
\end{aligned}
$$




\subsection{Asymptotic expansion}

We look for a solution of Equations (3.2) and (3.3) as an expansion of the small parameter $\epsilon_{a}$ :

$$
\begin{aligned}
& f=f^{(0)}+\epsilon_{a} f^{(1)}+\epsilon_{a}^{2} f^{(2)}+\ldots, \\
& \bar{\varepsilon}=\bar{\varepsilon}^{(0)}+\epsilon_{a} \bar{\varepsilon}^{(1)}+\epsilon_{a}^{2} \bar{\varepsilon}^{(2)}+\ldots,
\end{aligned}
$$

where we impose $\int_{\mathbb{R}^{3}} f^{(0)}(\mathbf{u} ; z, t) d \mathbf{u}=1$ and $\forall i \geq 1, \int_{\mathbb{R}^{3}} f^{(i)}(\mathbf{u} ; z, t) d \mathbf{u}=0$, without loss of generality. The zero ${ }^{\text {th }}$ order of the expansion for $f$ obeys the following Fokker-Planck equation:

$$
\frac{\partial f^{(0)}}{\partial t}=\frac{\partial}{\partial u_{j}}\left[\frac{C_{1}}{2} \bar{\omega}^{(0)} u_{j} f^{(0)}\right]+\frac{C_{0}}{2} \bar{\varepsilon}^{(0)} \frac{\partial^{2} f^{(0)}}{\partial u_{j} \partial u_{j}}
$$

with $\bar{\omega}^{(0)}=\bar{\varepsilon}^{(0)} / \bar{k}^{(0)}$. Besides, the zero ${ }^{\text {th }}$ order kinetic energy and its dissipation evolve according to:

$$
\frac{\partial \bar{k}^{(0)}}{\partial t}=-\bar{\varepsilon}^{(0)} \quad, \quad \frac{\partial \bar{\varepsilon}^{(0)}}{\partial t}=-C_{\varepsilon_{2}} \frac{\bar{\varepsilon}^{(0)^{2}}}{\bar{k}^{(0)}} .
$$

From these equations, it can be shown that the long-time solution of the PDF $f^{(0)}$ is an isotropic Gaussian of variance $\sigma^{2}=2 \bar{k}^{(0)} / 3$. We hereafter assume that time is large enough so that this long-time solution is reached. Equivalently, we can assume that the initial condition of $f$ is an isotropic Gaussian and that we look at the development of a perturbation around this initial state. In either case, we hereafter consider that:

$$
f^{(0)}(\mathbf{u} ; z, t)=\frac{e^{-\frac{u_{i} u_{i}}{2 \sigma^{2}}}}{\left(2 \pi \sigma^{2}\right)^{3 / 2}} \quad \text { with } \quad \sigma^{2}=\frac{2}{3} \bar{k}^{(0)}
$$

The derivation of the first order of the expansion is detailed in appendix A. The central result is that, for large times, $f^{(1)}$ takes the following form:

$$
f^{(1)}=C_{g} \frac{\sigma}{\bar{\omega}} \frac{\partial_{z} \sigma^{2}}{\sigma^{2}} \frac{u_{1}}{\sigma}\left(5-\frac{u_{i} u_{i}}{\sigma^{2}}\right) f^{(0)} \quad \text { with } C_{g}=\frac{1}{3 C_{1}+2 C_{\varepsilon_{2}}-6}
$$

The first order perturbation $f^{(1)}$ does not contribute to the Reynolds stresses:

$$
{\overline{u_{i} u_{j}}}^{(1)}=0 .
$$

However, it yields the main contribution to the third order moments. From the previous formula, one has:

$$
{\overline{u_{i} u_{j} u_{k}}}^{(1)}=-2 C_{g} \frac{\sigma^{2}}{\bar{\omega}} \frac{\partial \sigma^{2}}{\partial z}\left(\delta_{1 i} \delta_{j k}+\delta_{1 j} \delta_{i k}+\delta_{1 k} \delta_{i j}\right) .
$$

In particular, the flux of kinetic energy is given by:

$$
{\overline{u_{i} k}}^{(1)}=-5 C_{g} \frac{\sigma^{2}}{\bar{\omega}} \frac{\partial \sigma^{2}}{\partial z} \delta_{i 1}
$$

The second order is not detailed here. It yields an anisotropic contribution to the Reynolds stresses and an even contribution to the PDF, with a dependency on the gradient of $\sigma^{2}$ and on its Laplacian. 


\subsection{Main result: approximate PDF solution in the weakly inhomogeneous regime}

\subsubsection{Main result}

We now collect the zero ${ }^{\text {th }}$ and first orders of the expansion. Besides, we also go back to the original spatial coordinate $x_{1}=\epsilon_{a} z$. Then, we obtain that:

$$
\text { For } \frac{\ell}{L} \ll 1 \quad, \quad f\left(\mathbf{u} ; x_{1}, t\right)=\left[1+\sqrt{2 / 3} C_{g} \ell \frac{1}{\bar{k}} \frac{\partial \bar{k}}{\partial x_{1}} \frac{u_{1}}{\sqrt{2 \bar{k} / 3}}\left(5-\frac{u_{i} u_{i}}{2 \bar{k} / 3}\right)\right] \frac{e^{-u_{i} u_{i} /(4 \bar{k} / 3)}}{(4 \pi \bar{k} / 3)^{3 / 2}}
$$

where $\bar{k}$ is solution of a $\bar{k}-\bar{\varepsilon}$-like system:

$$
\text { For } \begin{aligned}
\frac{\ell}{L} \ll 1 \quad, \quad \frac{\partial \bar{k}}{\partial t} & =\frac{\partial}{\partial x_{1}}\left(C_{k} \frac{\bar{k}^{2}}{\bar{\varepsilon}} \frac{\partial \bar{k}}{\partial x_{1}}\right)-\bar{\varepsilon}, \\
\frac{\partial \bar{\varepsilon}}{\partial t} & =\frac{\partial}{\partial x_{1}}\left(C_{\varepsilon} C_{k} \frac{\bar{k}^{2}}{\bar{\varepsilon}}\left(\frac{\partial \bar{\varepsilon}}{\partial x_{1}}-\bar{k} \frac{\partial \bar{\omega}}{\partial x_{1}}\right)\right)-C_{\varepsilon_{2}} \bar{\omega} \bar{\varepsilon}
\end{aligned}
$$

with:

$$
C_{k}=\frac{20}{9} C_{g}=\frac{20}{9\left(3 C_{1}+2 C_{\varepsilon_{2}}-6\right)} .
$$

To obtain these expressions, we used the relations $\ell=\bar{k}^{3 / 2} / \bar{\varepsilon}=\sqrt{3 / 2} \sigma / \bar{\omega}$ and $\sigma^{2}=2 \bar{k} / 3$. We also injected relation (3.8) into the evolution Equations (2.4)-(2.5) of $\bar{k}$ and $\bar{\varepsilon}$.

Equations (3.9)-(3.11) are the main result of this work. They show that, in the weakly inhomogeneous regime, the simplified Langevin PDF model behaves as a standard $\bar{k}-\bar{\varepsilon}$ model. In particular, turbulent transport is given on first order by a diffusion term which coefficient depends explicitly on two model constants: $C_{1}$ and $C_{\varepsilon_{2}}$. The physical implications of this finding will be discussed in section 5 .

\subsubsection{Comment on the domain of validity of the main result}

The asymptotic expansion of the PDF eq. (3.9) and the ensuing $\bar{k}-\bar{\varepsilon}$ system (3.10)-(3.11) are valid in weakly inhomogeneous turbulence, i.e. for $\ell / L \ll 1$. Let us assume that this condition is indeed verified on the whole spatial domain. Then, when $C_{\varepsilon}=1$, the $\bar{k}-\bar{\varepsilon}$ system (3.10)-(3.11) admits a self-similar solution, first found by Barenblatt \& co-workers [9] and later by Cherfils \& Harrison [10]. This solution is reached for asymptotically large times for arbitrary initial conditions. For initial conditions that are compatible with the self-similar solution, the self-similar regime is immediately reached. It is given by:

$$
\begin{gathered}
\bar{k}\left(x_{1}, t\right)=\bar{k}_{0}\left(1+t / \tau_{0}\right)^{-2+2 \beta}\left(1-\left[x_{1} / \Lambda(t)\right]^{2}\right) \quad, \quad \varepsilon\left(x_{1}, t\right)=\bar{\varepsilon}_{0}\left(1+t / \tau_{0}\right)^{-3+2 \beta}\left(1-\left[x_{1} / \Lambda(t)\right]^{2}\right) \\
\text { with } \Lambda(t)=\Lambda_{0}\left(1+t / \tau_{0}\right)^{\beta} \quad \text { and } \quad \beta=\frac{2 C_{\varepsilon_{2}}-3}{3\left(C_{\varepsilon_{2}}-1\right)} .
\end{gathered}
$$

The values of $\bar{k}$ and $\bar{\varepsilon}$ at $t=0$ and $x_{1}=0$ are related to the two free parameters defining the initial length of the profile $\Lambda_{0}$ and the initial turbulent time $\tau_{0}$ :

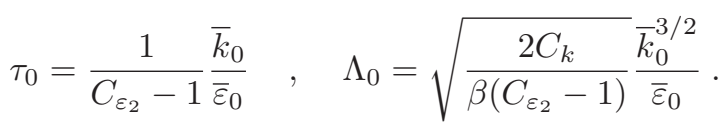


From this analytical solution, we can compute the small parameter on which the asymptotic expansion is based: $\ell / L=\left(\bar{k}^{3 / 2} / \bar{\varepsilon}\right)\left(\partial_{x_{1}} \bar{k} / \bar{k}\right)$. We find that:

$$
\frac{\ell}{L}=\sqrt{2 \beta\left(C_{\varepsilon_{2}}-1\right) / C_{k}} \frac{x_{1} / \Lambda}{\sqrt{1-\left(x_{1} / \Lambda\right)^{2}}} .
$$

This ratio is equal to 0 at the center of the mixing zone and grows to infinity at the edges of the mixing zone. The condition $\ell / L \ll 1$ is met only for:

$$
\frac{\left|x_{1}\right|}{\Lambda} \ll \frac{x_{10}}{\Lambda}=\frac{1}{\sqrt{1+2 \beta\left(C_{\varepsilon_{2}}-1\right) / C_{k}}}
$$

where the value of $x_{10} / \Lambda$ is on the order of 1 .

As a result, we deduce that the initial hypothesis of this reasoning, according to which the weakly inhomogeneous limit $\ell / L \ll 1$ is met on the whole spatial domain, cannot be fulfilled. Because of this, differences will exist between the solution of the actual $f$ and $\bar{\varepsilon}$ equations (2.1)-(2.5) and the solution of the approximate system (3.9)-(3.11), when applied to the whole domain, instead of being restricted to is domain of validity.

The question that follows is whether these differences will affect significantly the overall dynamical evolution of the mixing zone or not. In particular, it is important to know whether the evolution of $\bar{k}$ and $\bar{\varepsilon}$ obtained from the initial equations (2.1)-(2.5) differs substantially or not from the evolution of the approximate $\bar{k}-\bar{\varepsilon}$ model (3.10)-(3.11) applied to the whole domain. If the two remain close, the weakly inhomogeneous asymptotic expansion will not only yield a local approximation of the PDF and its moments, but also provide a global approximation of the evolution of the mixing zone.

The answer to these questions cannot be determined beforehand with the present analysis. Instead, numerical simulations are performed in the next section to give elements of discussion.

\section{NumericAl Simulations}

The purpose of the simulations is two-fold. First, when the parameter for the asymptotic expansion is small (eq. (3.1)), we want to corroborate numerically the analytical asymptotic expansion of the PDF (eq. (3.9)). Second, we would like to check whether or not the actual evolution of the mixing zone given by Equations $(2.1)$ and (2.5) remains close to the approximate evolution given by System (3.9)-(3.11). Indeed, as noted in section 3.3.2, the weakly inhomogeneous condition (3.1) and the ensuing approximation (3.9)-(3.11) cannot apply on the whole spatial domain, but fail close to the mixing zone edges.

To achieve those objectives, we propose two types of numerical reference solutions for the PDF equations (2.1) and (2.5). On the one hand, we consider a Eulerian Monte Carlo (EMC) solver. EMC methods have been introduced for scalar PDFs in [11,12] and have been extended to include the velocity field in [13]. They are described in appendix B. On the other hand, we use a direct deterministic solver based on finite volume approximations, described in appendix C. Given the high number of dimensions of Equation (2.1), the computational cost of a deterministic method is too expensive. Hence, we decide to apply the deterministic method to a simplified version of Equations (2.1) and (2.5). This simplified system is described in section 4.2.

In all simulations, we will restrict our attention to the case $C_{\epsilon}=1$ which allows for the analytical solution (3.12).

\subsection{Eulerian Monte Carlo simulations}

First, we solve Equations (2.1) and (2.5) with the Eulerian Monte Carlo (EMC) solver which principle is detailed in appendix B. The parameters of the simulation are the following. The computational domain

$\left[x_{\min }, x_{\max }\right]$ is set to $[-80,80]$. It is discretized with $N_{x}=256$ points. The number of stochastic fields is set to 
$N_{f}=16000$. The initial conditions are set according to the expected solution (3.12):

$$
\bar{k}\left(x_{1}, t=0\right)=\bar{k}_{0}\left(1-\left[\frac{x_{1}}{\Lambda_{0}}\right]^{2}\right) \quad, \quad \bar{\varepsilon}\left(x_{1}, t=0\right)=\bar{\varepsilon}_{0}\left(1-\left[\frac{x_{1}}{\Lambda_{0}}\right]^{2}\right),
$$

where we set the values $\Lambda_{0}=10$ and $\bar{k}_{0}=1.5$ and where the values of $\tau_{0}$ and $\bar{\varepsilon}_{0}$ are given by Formula (3.13). Two calculations are done: one with $C_{1}=4.15$ and one with $C_{1}=1.8$. For $C_{1}=1.8$, one has $\tau_{0}=2.0$ and $\bar{\varepsilon}_{0}=0.84$ and for $C_{1}=4.15$, one has $\tau_{0}=3.6$ and $\bar{\varepsilon}_{0}=0.47$.

\subsubsection{Validity of the asymptotic expansion}

The first point we examine is whether the validity conditions of the asymptotic expansion are met and, if it is the case, whether the asymptotic expansion is indeed obtained. Figure 2 shows the profiles of $\ell / L$ at $t / \tau_{0}=10$. It can be seen that this ratio is $\ell / L=\mathcal{O}(1)$ at $1 / 2 \lesssim x / L_{k}(t) \lesssim 1$, and falls close to zero at $x / L_{k}(t)=0$. It can also be seen that $\ell / L$ slowly goes back to zero at the edges of the mixing zone for $\ell / L_{k}(t) \geq 1$. The length $L_{k}$ is defined as:

$$
L_{k}(t)=\frac{3}{4} \frac{\int \bar{k}\left(x_{1}, t\right) d x_{1}}{\max _{x_{1} \in \mathbb{R}}\left(\bar{k}\left(x_{1}, t\right)\right)} .
$$

It is equal to $\Lambda(t)$ when $\bar{k}$ and $\bar{\varepsilon}$ obey the self-similar solution (3.12). While other definitions are possible, we will hereafter consider that $L_{k}$ defines the size of the mixing zone: $L_{T Z}=L_{k}$.

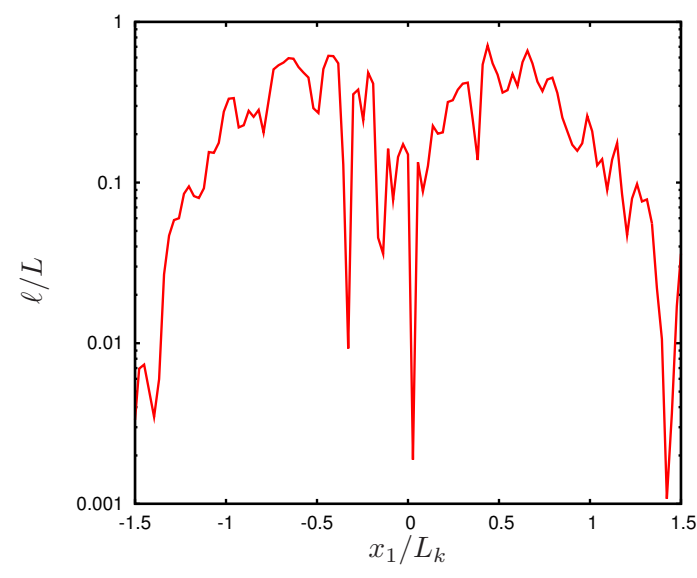

(a) $C_{1}=1.8$

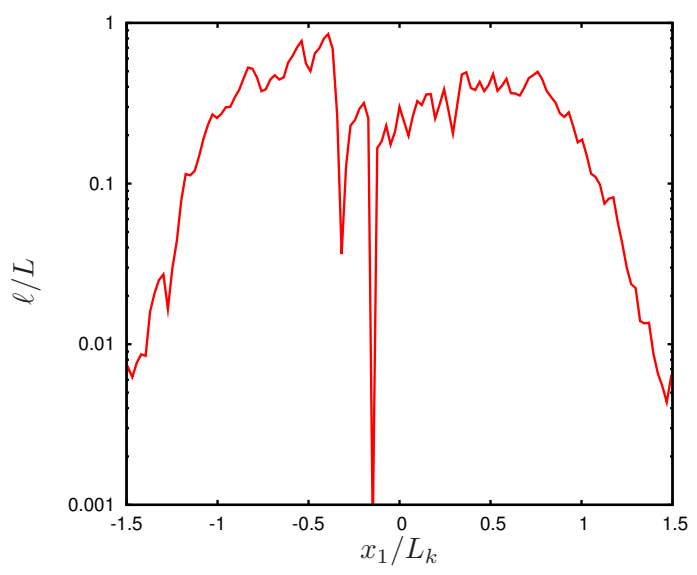

(b) $C_{1}=4.15$

Figure 2. $\ell / L$ as a function of $x_{1} / L_{k}$ at time $t / \tau_{0}=10$

The estimate of the PDF obtained from the EMC code is too noisy to be compared directly to the analytical form (3.9). Direct comparisons will be made with the deterministic code. However, we can compare predicted and computed moments. In particular, Figure 3 shows a comparison of the third order moment $\overline{u_{1} k}$ against its predicted value given by Equation (3.8). More precisely, Figure 3 shows the quantity:

$$
\Delta F=\frac{\left|\overline{u_{1} k}+C_{k} \frac{\bar{k}^{2}}{\bar{\varepsilon}} \frac{\partial \bar{k}}{\partial x_{1}}\right|}{\bar{k}^{3 / 2}}
$$

The main observation is that predicted and computed third order moments are close to one another in the 


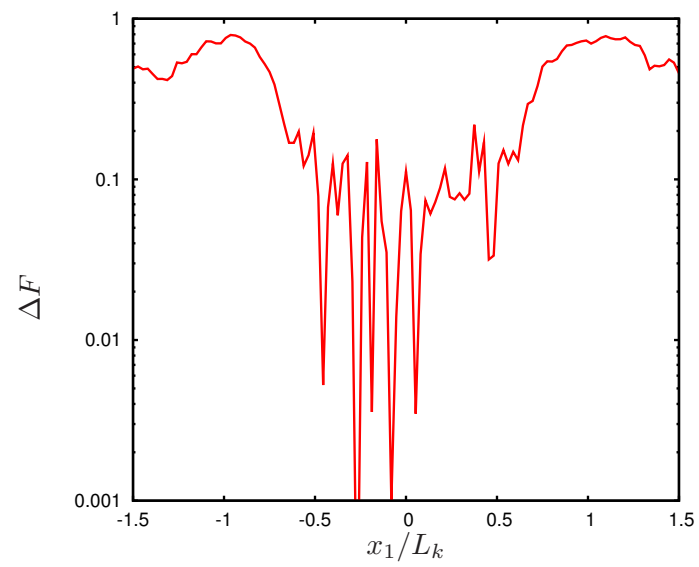

(a) $C_{1}=1.8$

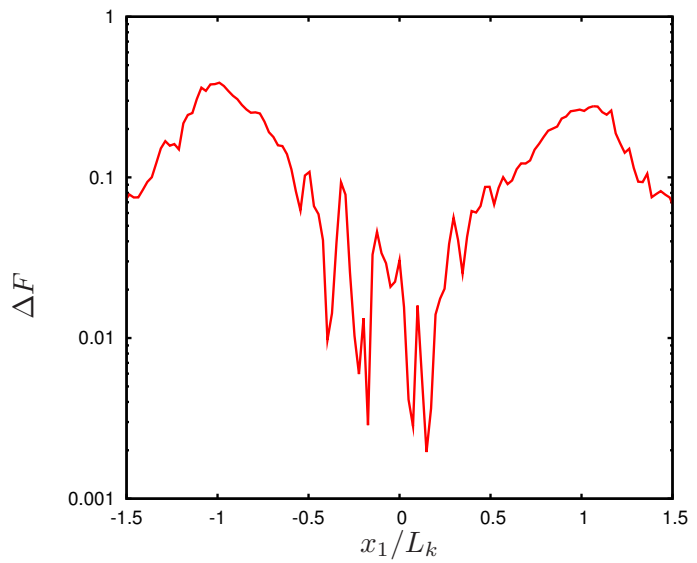

(b) $C_{1}=4.15$

Figure 3. $\Delta F$ as a function of $x_{1} / L_{k}$ at time $t / \tau_{0}=10$

central part of the mixing zone, approximately for $\left|x_{1}\right| / L_{k} \leq 0.5$. Their difference is maximum for $\left|x_{1}\right| / L_{k} \approx 1$ and decreases slowly beyond this point. These observations are in agreement with the expected domain of validity of the asymptotic expansion, as commented from Figure 2.

\subsubsection{Evolution of the mixing zone}

We now turn our attention to the question asked at the end of section 3.3.2: whether the evolution of $\bar{k}$ and $\bar{\varepsilon}$ differs substantially or not from the analytical prediction (3.12). To do so, we first assess the self-similarity of the solution. In addition to $L_{k}$, we introduce the following two parameters:

$$
\bar{k}_{\max }(t)=\max _{x_{1} \in \mathbb{R}}\left(\bar{k}\left(x_{1}, t\right)\right) \quad, \quad \bar{\varepsilon}_{\max }(t)=\max _{x_{1} \in \mathbb{R}}\left(\bar{\varepsilon}\left(x_{1}, t\right)\right) .
$$

We first consider the time evolutions of the three parameters $\bar{k}_{\text {max }}(t), \bar{\varepsilon}_{\text {max }}(t)$ and $L_{k}(t)$ and compare them against their predicted values given by the self-similar solution (3.12). To this end, we introduce the three ratios $R_{k}, R_{\varepsilon}$ and $R_{L}$ defined by:

$$
R_{k}=\frac{\bar{k}_{\max }}{\bar{k}_{0}\left(1+t / \tau_{0}\right)^{-2+2 \beta}} \quad, \quad R_{\varepsilon}=\frac{\bar{\varepsilon}_{\max }}{\bar{\varepsilon}_{0}\left(1+t / \tau_{0}\right)^{-3+2 \beta}} \quad, \quad R_{L}=\frac{L_{k}}{\Lambda_{0}\left(1+t / \tau_{0}\right)^{\beta}} .
$$

If the solution remains close to the self-similar solution (3.12), then $R_{k}, R_{\varepsilon}$ and $R_{L}$ should become independent of time. Besides, given that the initial condition was chosen close to a self-similar solution, one should have $R_{k}=R_{\varepsilon}=R_{L} \approx 1$. A strict equality is not expected since the asymptotic expansion is not valid on the whole domain and since the initial condition is not fully coherent with the self-similar state. In particular, the initial PDF is a Gaussian, whereas the self-similar PDF deviates from Gaussianity.

The three ratios $R_{k}, R_{\varepsilon}$ and $R_{L}$ are displayed in Figure 4. It can be seen that they indeed remain approximately constant and stay close to one for the two simulations respectively performed with $C_{1}=1.8$ and $C_{1}=4.15$.

We now consider the ratio $\bar{k} / k_{\max }$ taken at different times is displayed in Figure 5 as a function of $x_{1} / L_{k}$ and for two values of $C_{1}$. It can be seen that the respective profiles of the two ratios fall approximately on a single curve. This indicates that $\bar{k}$ is close to a self-similar state. Besides, the collapsed curves remain close to parabolas as predicted by solution (3.12). The main difference with this solution occurs at the edges of the turbulent zone 


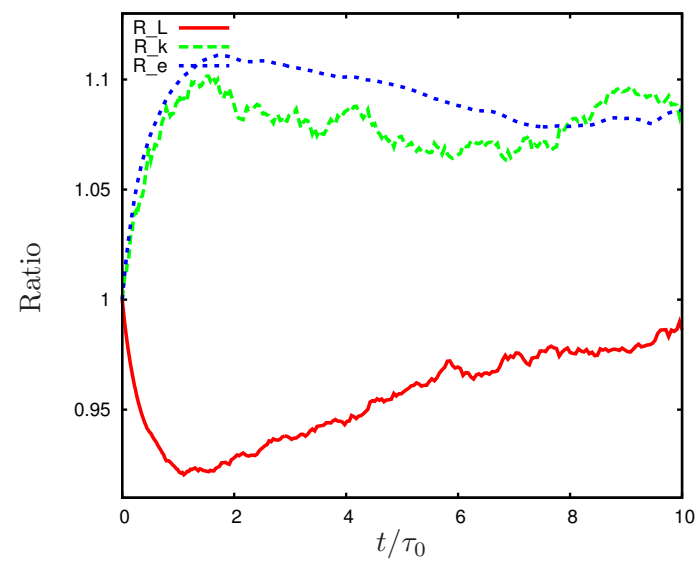

(a) $C_{1}=1.8$

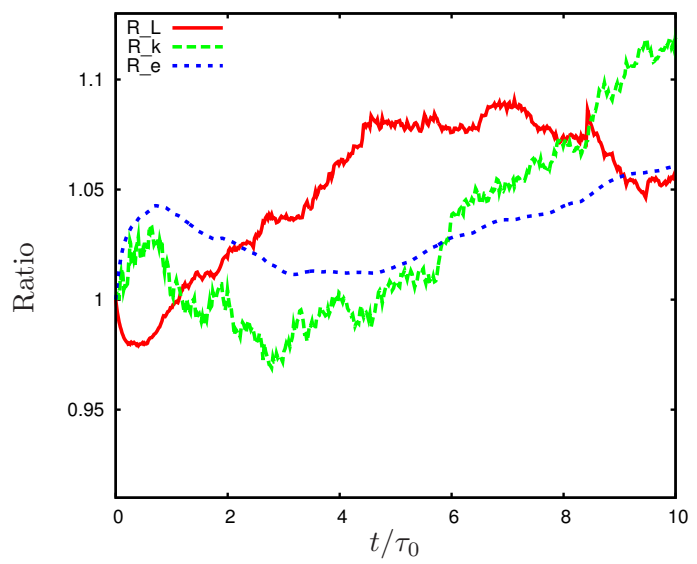

(b) $C_{1}=4.15$

Figure 4. Evolution of $R_{k}, R_{\varepsilon}$ and $R_{L}$ as a function of time.

: while Equation (3.12) predicts a compactly supported turbulent kinetic energy $\bar{k}$, the simulation yields a non-compact one. Compactly supported initial conditions indeed lead to compactly supported solutions for the system (3.10)-(3.11). The departure of the simulation results from the self-similar compactly supported solution can be explained by the high values of the expansion parameter $\ell / L$ at the edges of the TZ. At these locations, the asymptotic expansion ceases to be valid and $\bar{k}$ and $\bar{\varepsilon}$ cease to be governed by Equations (3.10)-(3.11).

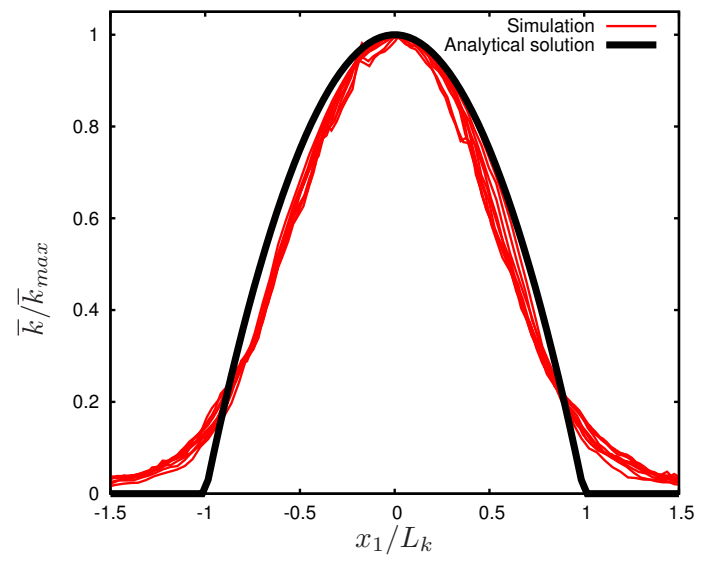

(a) $C_{1}=1.8$

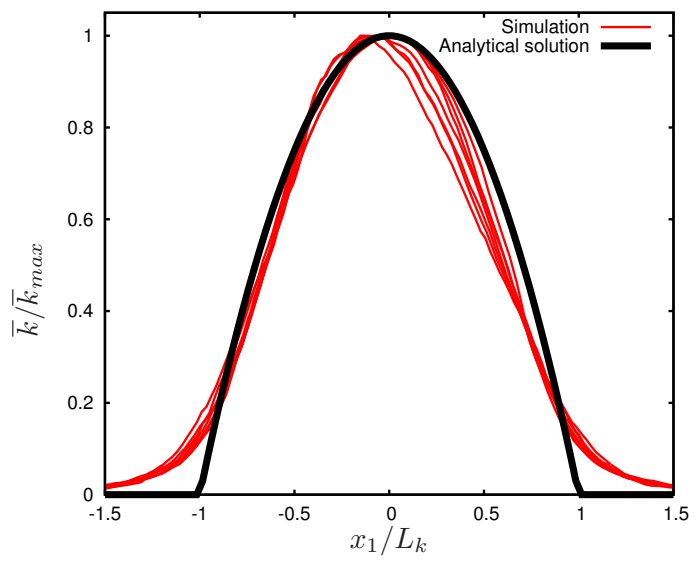

(b) $C_{1}=4.15$

FiguRE $5 . \bar{k} / \bar{k}_{\text {max }}$ as a function of $x_{1} / L_{k}$ at different times from $t / \tau_{0}=2$ to $t / \tau_{0}=10$

The existence and properties of the self-similar solution arise in part from the approximation of the flux of kinetic energy given by Formula (3.8). This approximation has been checked directly in Figure 3. Here, we would like make an additional comparison against the self-similar prediction corresponding to Equation (3.12). 
To this end, we introduce the non-dimensional flux :

$$
F^{*}\left(x_{1}, t\right)=\frac{\overline{u_{1} k}}{\bar{k}_{\max }^{3 / 2} \sqrt{2 \beta C_{k}\left(C_{\varepsilon_{2}}-1\right)}} .
$$

According to Formula (3.8) and (3.12), $F^{*}$ should be equal to $x_{1} / L_{k}\left(1-\left[x_{1} / L_{k}\right]^{2}\right)$. The comparison between the two functions is displayed at Figure 6 for different times. It can be seen that both simulation and prediction are in good agreement in the central part of the mixing zone, for $x_{1} / L_{k} \in[-0.5,0.5]$. Outside, the gradient diffusion assumption ceases to be relevant: the predicted flux of kinetic energy becomes much smaller than the simulated flux. This observation is consistent with the one made on the non-compactness of the $\bar{k}-\bar{\varepsilon}$ supports observed in Figure 5.

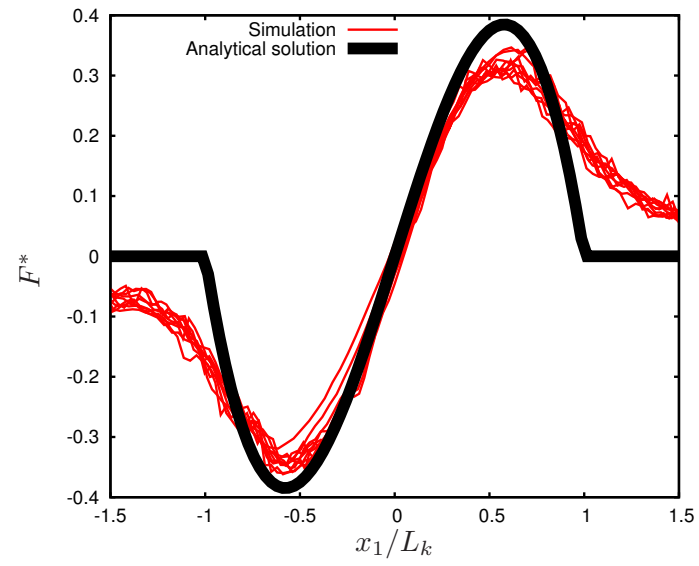

(a) $C_{1}=1.8$

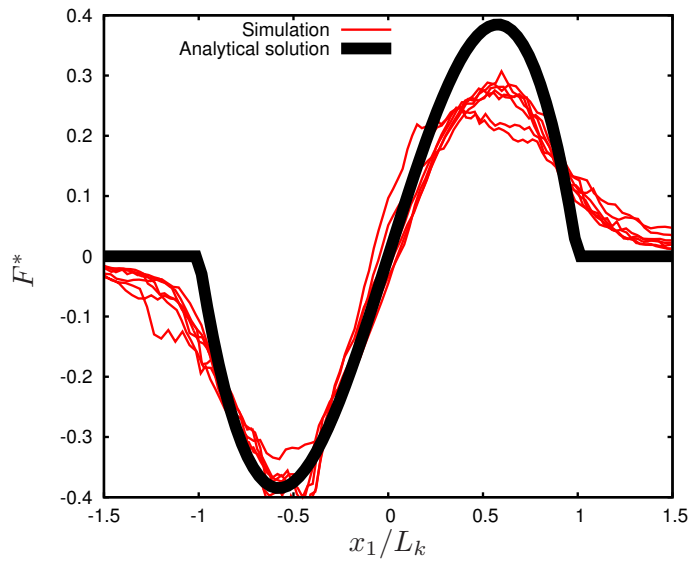

(b) $C_{1}=4.15$

FiguRE 6. $F^{*}$ as a function of $x_{1} / L_{k}$ at different times from $t / \tau_{0}=2$ to $t / \tau_{0}=10$.

As a conclusion, the simulation results appear to be in good agreement with the self-similar solution (3.12) of the $\bar{k}-\bar{\varepsilon}$ system (3.10)-(3.11). Thus, these simulations suggest that the asymptotic expansion of the PDF eq. (3.9) and the ensuing $\bar{k}-\bar{\varepsilon}$ system (3.10)-(3.11) offer a good estimate of the overall evolution of the mixing zone given by the full PDF system (2.1) and (2.5).

\subsection{Deterministic finite volume simulations}

The Eulerian Monte Carlo method has allowed to study some properties of the second and third order moments of the velocity field. However, its intrinsic noise is too high to directly study the PDF. To circumvent this deficiency, we propose to use a deterministic solver described in appendix C.

\subsubsection{Simplification of System (2.1)-(2.5)}

The basic configuration studied hereafter is the same TZ configuration introduced in section 2 and governed by Equations (2.1)-(2.5). As explained above, Equations (2.1)-(2.5) have a high number of dimensions : 1 in time and 4 in velocity and physical space. The computational cost of a deterministic method is too expensive so that we propose to simplify these equations in order to reduce their dimensionality. More precisely, we focus on the marginal PDF $f_{1}$ of $u_{1}$. By integrating Equation (2.1) over $u_{2}$ and $u_{3}$, one obtains that $f_{1}$ evolves as:

$$
\frac{\partial f_{1}}{\partial t}+u_{1} \frac{\partial f_{1}}{\partial x_{1}}=-\frac{\partial}{\partial u_{1}}\left[\left(\frac{\partial \overline{u_{1}^{2}}}{\partial x_{1}}-\frac{C_{1}}{2} \bar{\omega} u_{1}\right) f_{1}\right]+\frac{C_{1}-1}{2} \bar{\varepsilon}^{*} \frac{\partial^{2} f_{1}}{\partial u_{1} u_{1}},
$$


where $\bar{\varepsilon}^{*}=\frac{2}{3} \bar{\varepsilon}$ is the dissipation of $\overline{u_{1}^{2}}$. This equation is closed provided the evolution of $\bar{\varepsilon}^{*}$ is known in terms of the statistics of $u_{1}$. This is not the case of Equation (2.5) which is related to $\bar{k}$. Hence, we propose to simplify this equation. Namely, we assume that the Reynolds stresses are strictly isotropic. Then, the turbulent frequency can be related to $\overline{u_{1}^{2}}$ according to: $\bar{\omega}=\bar{\varepsilon} / \bar{k}=\bar{\varepsilon}^{*} / \overline{u_{1}^{2}}$. Besides, we assume that $\overline{u_{1} u_{i} u_{j}}$ is also an isotropic tensor, which yields $\overline{u_{1} k}=3 \overline{u_{1}^{3}} / 2$. With these assumptions, one deduces from Equation (2.5) the following simplified evolution for $\bar{\varepsilon}^{*}$ :

$$
\frac{\partial \bar{\varepsilon}^{*}}{\partial t}+\frac{\partial}{\partial x_{1}}\left(C_{\varepsilon} \bar{\omega} \overline{u_{1}^{3}}\right)=-C_{\varepsilon_{2}} \bar{\omega} \bar{\varepsilon}^{*}
$$

Equations (4.1)-(4.2) are three dimensional and can be solved with the deterministic solver. They share the same properties as Equations (2.1)-(2.5) but present a slight variation in the weakly inhomogeneous limit. The limit of $f_{1}$ is, as expected, the integral of the limit of $f(3.9)$ over $u_{2}$ and $u_{3}$ :

$$
f_{1}\left(u_{1} ; x_{1}, t\right)=\frac{e^{-u_{1}^{2} /\left(2 \overline{u_{1}^{2}}\right)}}{\sqrt{2 \pi \overline{u_{1}^{2}}}}\left(1+C_{g}\left(\frac{1}{\sqrt{\overline{u_{1}^{2}}} \bar{\omega}} \frac{\partial \overline{u_{1}^{2}}}{\partial x_{1}}\right) \frac{u_{1}}{\sqrt{\overline{u_{1}^{2}}}}\left(3-\frac{u_{1}^{2}}{\overline{u_{1}^{2}}}\right)\right),
$$

However, the value of $\overline{u_{1}^{3}}$ is not given by Formula (3.7) but by:

$$
\overline{u_{1}^{3}}=-C_{k} \frac{\overline{u_{1}^{2}}}{\bar{\omega}} \frac{\partial \overline{u_{1}^{2}}}{\partial x_{1}}
$$

with $C_{k}=6 C_{g}$. The notation $C_{k}$ has been retained here because in the diffusion limit, $\overline{u_{1}^{2}}$ and $\bar{\varepsilon}^{*}$ obey a $\bar{k}-\bar{\varepsilon}$ like system similar to Equations (3.10)-(3.11). The solution of this system is then obtained directly from Equations (3.12) by replacing $\bar{k}$ by $\overline{u_{1}^{2}}$ and $\bar{\varepsilon}$ by $\bar{\varepsilon}^{*}$.

\subsubsection{Set-up}

The computational domain is defined by $\left[x_{\min }, x_{\max }\right]=[-30,30]$ and $\left[u_{\min }, u_{\max }\right]=[-6,6]$. It is discretized with $\left(n_{x}, n_{v x}\right)=256^{2}$ points and the time step is set to $d t=2 \cdot 10^{-3}$. The initial conditions are set according to

$$
\overline{u_{1}^{2}}\left(x_{1}, t=0\right)=\bar{k}_{0}\left(1-\left[\frac{x_{1}}{\Lambda_{0}}\right]^{2}\right)+\bar{k}_{\text {min }} \quad, \quad \bar{\varepsilon}^{*}\left(x_{1}, t=0\right)=\bar{\varepsilon}_{0}\left(1-\left[\frac{x_{1}}{\Lambda_{0}}\right]^{2}\right),
$$

where $\bar{k}_{0}=1$ and $\Lambda_{0}=10$ and where the values of $\tau_{0}$ and $\bar{\varepsilon}_{0}$ are given by Formula (3.13). The additional parameter $\bar{k}_{\text {min }}$ is set to $\bar{k}_{\text {min }}=10^{-2}$. It is required because Diracs cannot be represented in a deterministic method. They are here replaced by a Gaussian with a sufficiently small variance for the PDF to approximate a Dirac, and sufficiently large to obtain a numerical resolution of the PDF with a reasonable number of velocity points. The coefficient of the model is set to $C_{1}=2.73$, in order to recover $C_{k}=1$.

\subsubsection{Validity of the asymptotic expansion}

The value of the small expansion parameter stabilizes at $\ell / L=5.10^{-3}$ at the center of the $\mathrm{TZ}$, and $\ell / L=0.8$ at the edge of the TZ. As expected, the validity range of the expansion is therefore not verified a posteriori at all points of the domain, but only in the bulk of the mixing zone. This is similar to what was observed in the EMC simulation.

Then, we can analyse the anisotropic odd part of the PDF, with respect to the analytical one (denoted as $\epsilon_{a} f^{1}$ in Section 3.2). The comparison between the numerical and analytical PDF is shown in Figure 7, respectively at the center and at the edge of the TZ. It can be seen that the predicted and simulated PDF shapes are close to one another. This gives confidence in the asymptotic expansion derived in section 3.2. 


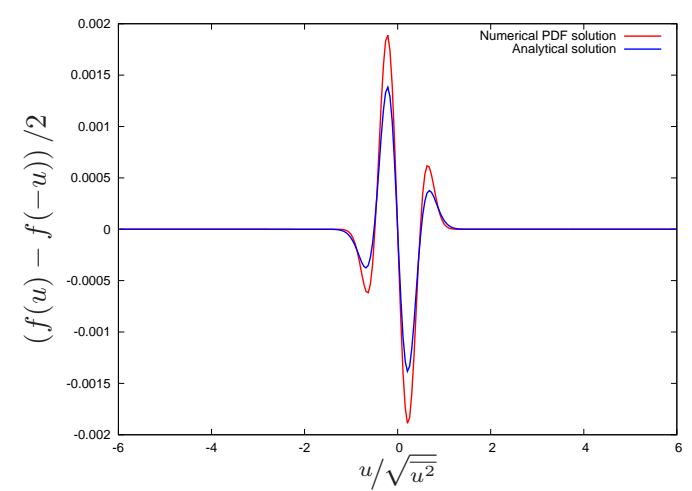

(a) PDF at the TZ center

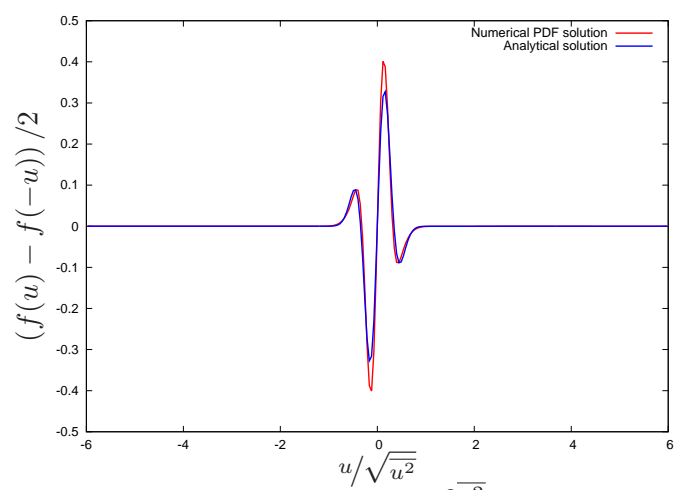

(b) PDF at the point where $\frac{\partial \overline{u^{2}}}{\partial x}$ is maximum

Figure 7. Odd part of the PDF. Comparison between the numerical PDF and the solution obtained from the asymptotic development, at $t / \tau_{0}=5$.

\subsubsection{Evolution of the mixing zone}

For the TZ configuration described in section 4.2, we first compare the diffusion solution (3.12), and the numerical solution of Equations (4.1)-(4.2). In Figures 8(a) and 8(b), we observe a good agreement between the numerical and the analytical solutions, for the second and third moments of the PDF. Moreover, in Figure 9, the self-similarity of the solution is checked, with respect to the quantities $R_{k}, R_{\epsilon}$ and $R_{L}$ (that are defined in Section 4.1.2). This shows that the PDF solution operates close to the diffusion regime, for which asymptotic PDF solutions have been derived in Section 3.2.

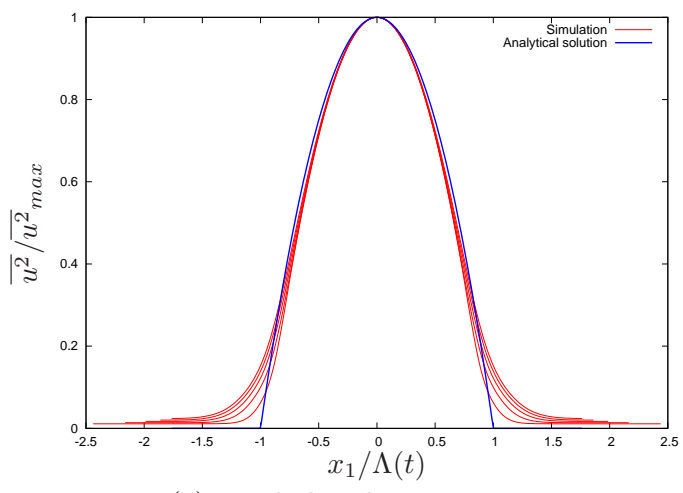

(a) Turbulent kinetic energy

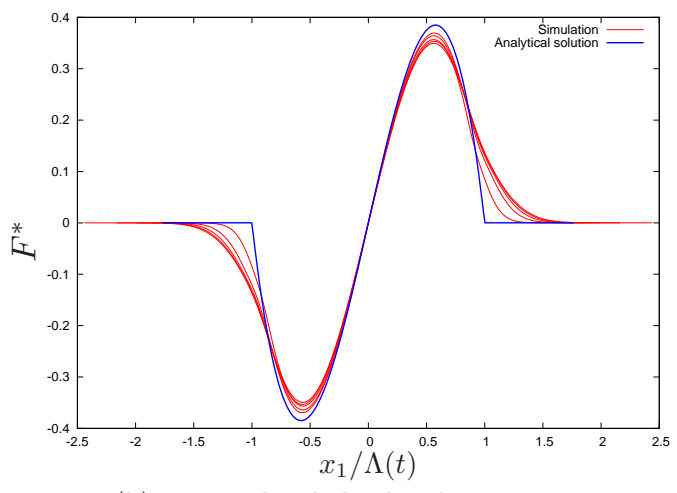

(b) Normalized third order moment

Figure 8. Comparison between Barenblatt analytical solution (3.12) and the numerical PDF solution from $t / \tau_{0}=1$ to $t / \tau_{0}=5$. The TZ support increases as time increases.

\section{Discussion AND CONCLUSIONS}

In section 3, we showed that, in the weakly inhomogeneous limit, the simplified Langevin model (SLM) gives rise to diffusion approximation for turbulent transport and behaves as a standard $\bar{k}-\bar{\varepsilon}$ model. In section 4 , 


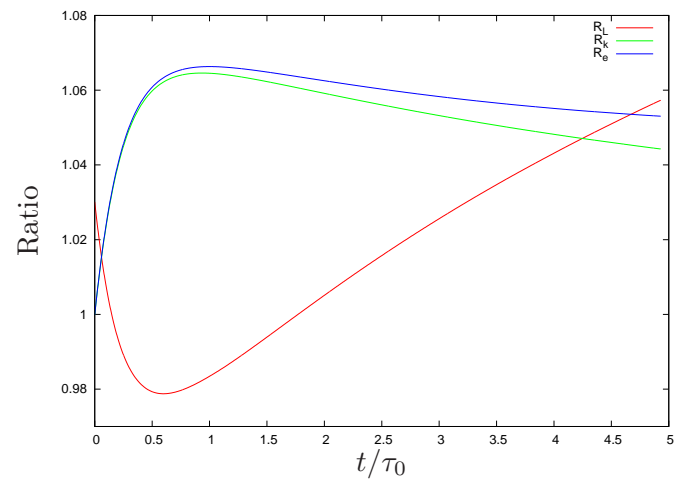

Figure 9. Evolution of $R_{k}, R_{\epsilon}$, and $R_{L}$ as a function of time.

we performed numerical simulations of a turbulent mixing zone and showed that the weakly inhomogeneous limit and the diffusion approximation were relevant to describe the diffusion and decay of turbulence in this configuration. These results have been obtained for the SLM. However, they should also apply to a broader class of Langevin models, including most of those described in $[2,3]$ and called Generalized Langevin models (GLM). Indeed, in the turbulent mixing zone configuration, these models only differ from the SLM by correction due the anisotropy tensor. In the weakly inhomogeneous expansion, this quantity is of order 2. Consequently, it will not modify the orders 0 and 1 on which the present discussion is based.

The results obtained in sections 3 and 4 raise a number of questions concerning the way turbulent transport is effectively modelled in Langevin PDF models. First, the transport of kinetic energy is given on first order by a gradient diffusion approximation. The corresponding diffusion coefficient $C_{k}$ is found to depend explicitly on two model constants: $C_{1}$ and $C_{\varepsilon_{2}}$. We recall that the constant $C_{\varepsilon_{2}}$ is set in order to reproduce the correct decay of kinetic energy in homogeneous isotropic turbulence. As for the constant $C_{1}$, it is set in order to specify the decay of the anisotropy tensor $b_{i j}=R_{i j}-2 \bar{k} / 3 \delta_{i j}$ in homogeneous turbulence. Hence, one is faced with an apparent contradiction : the coefficient controlling turbulent transport in Langevin PDF methods is set by observations and reasonings made in homogeneous turbulence, which by definition is devoid of turbulent transport.

Second, the value of $C_{1}$ varies in the literature and so does the value of the diffusion coefficient $C_{k}$. For $C_{1}=1.8$, one has $C_{k}=0.7$ and for $C_{1}=4.15$, one has $C_{k}=0.22$. These values have to be compared with the usual value retained in $\bar{k}-\bar{\varepsilon}$ models $C_{k}^{\bar{k}-\bar{\varepsilon}}=0.15-0.22$. Thus, if one wants to obtain results close to a standard $\bar{k}-\bar{\varepsilon}$ model in the diffusion-dissipation regime, one should rather choose a value of $C_{1}=4.15$. However, as explained in section 2, higher values of $C_{1}$ are usually associated with simpler models discarding the rapid contribution of the pressure gradient. For more realistic models, it is the value $C_{1}=1.8$ which is relevant. Hence, one is left to choose between a value of $C_{1}$ that captures correctly turbulent transport and a value that is compatible with the presence of a rapid pressure model. In addition to the first comment, this second remark tends to indicate that the definition of $C_{1}$ and the term it controls in the simplified Langevin model is overloaded. It looks as if the $C_{1}$ term in Equation (2.1) had to represent two distinct physical mechanisms: return to isotropy and turbulent transport.

Finally, a last remark must be made. While the Langevin PDF and $\bar{k}-\bar{\varepsilon}$ models behave alike in the diffusion limit, there is still a fundamental difference between the two. In the $\bar{k}-\bar{\varepsilon}$ model, the gradient diffusion term models turbulent advection and also turbulent transport by the pressure: $-C_{k}^{\bar{k}-\varepsilon} \frac{\bar{k}^{2}}{\bar{\varepsilon}} \partial_{x_{i}} \bar{k}=\overline{u_{i} k}+\overline{u_{i} p}$. By contrast, in the simplified Langevin PDF model, pressure transport is neglected. This can be seen in Equation (2.4) where only the flux of $\bar{k}$ appears. For the simplified Langevin model, one has: $-C_{k} \frac{\bar{k}^{2}}{\bar{\varepsilon}} \partial_{x_{i}} \bar{k}=\overline{u_{i} k}$. This relation could be justified if $\overline{u_{i} p}$ was negligible. However, this is not the case. In isotropic turbulence, one has exactly: $\overline{u_{i} p}=-2 / 5 \overline{u_{i} k}[14]$. Therefore, an important part of turbulent transport is missing in PDF models. 
Still, the fact that $\overline{u_{i} p}$ and $\overline{u_{i} k}$ are proportional allows for an effective definition of $C_{k}$ which accounts for the missing term and give an overall correct transport in the diffusion regime. In that case, the value of $\overline{u_{i} k}$ is overestimated by a factor $5 / 3 \approx 1.7$.

All these remarks point to some deficiencies in the way turbulent transport is represented in PDF models. We hope to address some of these deficiencies in a forthcoming paper.

\section{A. Derivation of the First order of the ASYMPtotic EXPANSION}

First of all, we note that $\bar{k}^{(1)}$ and $\bar{\varepsilon}^{(1)}$ obey a system of equations independent of higher orders:

$$
\frac{\partial \bar{k}^{(1)}}{\partial t}=-\bar{\varepsilon}^{(1)} \quad, \quad \frac{\partial \bar{\varepsilon}^{(1)}}{\partial t}=-C_{\varepsilon_{2}} \bar{\omega}^{(0)} \bar{\varepsilon}^{(0)}\left(2 \frac{\bar{\varepsilon}^{(1)}}{\bar{\varepsilon}^{(0)}}-\frac{\bar{k}^{(1)}}{\bar{k}^{(0)}}\right) .
$$

Zero being a particular solution of it, we can set, without loss of generality:

$$
\bar{k}^{(1)}=0 \text { and } \bar{\varepsilon}^{(1)}=0 .
$$

Then, the equation for $f^{(1)}$ becomes:

$$
\frac{\partial f^{(1)}}{\partial t}-\frac{\partial}{\partial u_{j}}\left[\frac{C_{1}}{2} \bar{\omega}^{(0)} u_{j} f^{(1)}\right]-\frac{C_{0}}{2} \bar{\varepsilon}^{(0)} \frac{\partial^{2} f^{(0)}}{\partial u_{j} \partial u_{j}}=-u_{1} \frac{\partial f^{(0)}}{\partial z}-\frac{\partial}{\partial u_{1}}\left[\frac{\partial \sigma^{2}}{\partial z} f^{(0)}\right] .
$$

A particular form $f^{(1)}=f^{(0)} \frac{u_{1}}{\bar{\omega}^{(0)}} g\left(\|\boldsymbol{u}\|^{2} / \sigma^{2}\right) \frac{1}{\sigma^{2}} \frac{\partial \sigma^{2}}{\partial z}$ is injected in this equation (we recall that $\sigma^{2}=2 \bar{k}^{(0)} / 3$ ). We find that the function $g(y)$ is solution of the following second order ODE:

$$
2 y \frac{d^{2} g}{d y^{2}}(y)+(5-y) \frac{d g}{d y}(y)-\frac{C_{\varepsilon_{2}}+C_{1} / 2-2-\Gamma}{C_{1}-1} g(y)=-\frac{1}{2} \frac{5-y}{C_{1}-1},
$$

where $\Gamma=\left[\frac{1}{\bar{\omega}^{(0)}} \frac{\partial \bar{\omega}^{(0)}}{\partial z}\right] /\left[\frac{1}{\sigma^{2}} \frac{\partial \sigma^{2}}{\partial z}\right]$. The solution of this equation is:

$$
g(y)=\frac{5-y}{3 C_{1}+2 C_{\varepsilon_{2}}-6-2 \Gamma}
$$

so that we obtain:

$$
f^{(1)}=\widetilde{C}_{g} \frac{\sigma}{\bar{\omega}} \frac{\partial_{z} \sigma^{2}}{\sigma^{2}} \frac{u_{1}}{\sigma}\left(5-\frac{u_{i} u_{i}}{\sigma^{2}}\right) f^{(0)} \quad \text { with } \widetilde{C}_{g}=\frac{1}{3 C_{1}+2 C_{\varepsilon_{2}}-6-2 \Gamma} .
$$

The evolution of $\Gamma$ is given by: $\frac{\partial \Gamma}{\partial t}=\bar{\omega}^{(0)} \Gamma^{2}-\left(C_{\varepsilon_{2}}-1\right) \bar{\omega}^{(0)} \Gamma$, which solution is:

$$
\Gamma=\frac{\Gamma_{0}\left(C_{\varepsilon_{2}}-1\right)}{\Gamma_{0}+\left(C_{\varepsilon_{2}}-1-\Gamma_{0}\right) e^{\left(C_{\varepsilon_{2}}-1\right) \int_{0}^{t} \bar{\omega}^{(0)}(s) d s}}
$$

with $\Gamma_{0}$ the initial condition of $\Gamma$. For physical reasons, we are only interested in solutions for which $\bar{k}^{(0)}$ and $\bar{\varepsilon}^{(0)}$ have approximately the same spatial profiles. In that case, $\Gamma_{0}$ remains small. In this work, we will more specifically restrict our attention to cases for which $\Gamma_{0}$ is smaller that $\left(C_{\varepsilon_{2}}-1\right)$. Then, $\Gamma$ tends to 0 , so that, for long times, we can neglect its contribution in the final expression of $\tilde{C}_{g}$. The PDF $f^{(1)}$ is then given by Equation (3.6). Note that the case $\Gamma_{0}$ larger than $\left(C_{\varepsilon_{2}}-1\right)$ would yield an infinite value of $\Gamma$ which is not physically relevant in the present context. The only other relevant case would be $\left(C_{\varepsilon_{2}}-1\right)=\Gamma_{0}$, which is a special limit not treated in this work. 


\section{B. Eulerian Monte Carlo solver}

Following [13], a statistical equivalence can be found between the PDF equation (2.1) and the following system of stochastic partial differential equations (SPDE):

$$
\begin{aligned}
& \frac{\partial r}{\partial t}+\frac{\partial}{\partial x_{1}}\left(r u_{1}\right)=0 \\
& \frac{\partial v_{i}}{\partial t} d t+v_{1} \frac{\partial v_{i}}{\partial x_{1}} d t=\frac{\partial R_{1 i}}{\partial x_{1}} d t-\frac{C_{1}}{2} \bar{\omega} v_{i} d t+\sqrt{C_{0} \bar{\varepsilon}} d W_{i}
\end{aligned}
$$

where $W_{i}$ are independent Wiener processes. More precisely, the weighted PDF:

$$
\tilde{f}=\frac{\overline{r\left(x_{1}, t\right) \delta\left(\boldsymbol{v}\left(x_{1}, t\right)-\mathbf{u}\right)}}{\bar{r}\left(x_{1}, t\right)}
$$

has an evolution equation identical to equation (2.1). Then provided the right boundary and initial conditions are chosen, system (B.1) will allow to solve PDF equation (2.1).

From a numerical point of view, the main difficulty lies in solving the advection part of the equation: $\frac{\partial v_{i}}{\partial t}+v_{1} \frac{\partial v_{i}}{\partial x_{1}}$. In the PDF equation the equivalent of this term corresponds to purely linear advection and this property should be preserved in the interpretation of the SPDEs. Thus, the latter should be interpreted as quasi-linear hyperbolic equations: characteristic curves of the SPDEs can cross, which results in a multivalued solutions. At points where the solution of the velocity is multivalued, the corresponding density also becomes a multivalued function. This kind of properties also arises in different contexts. See for instance [15].

Traditional numerical schemes which satisfy an entropy increase condition are not appropriate for the description of multivalued solutions. Instead, one can use a level-set based method as in [15]. Alternatively, given the stochastic nature of our problem, on can devise a variation on the Random Choice Method (RCM) [16]. The details of the derivation of these methods will be given in a forthcoming article. We only give here its final expression.

Space is discretized with a uniform mesh $x_{j}=j \Delta x, j=1 \cdots N_{x}$. Time is discretized on intervals $\left[t^{n}, t^{n+1}=\right.$ $\left.t^{n}+\Delta t\right] . N_{s}$ stochastic fields are solved simultaneously. They are each indexed by the superscript $(s)$. For the sake of simplicity, we will only use this index when necessary. For a given stochastic field, the numerical scheme is the following:

$$
\begin{aligned}
r_{j}^{n+1} & =r_{j}^{n}-\frac{\Delta t}{\Delta x}\left[\left|\left(v_{1}\right)_{j}^{n}\right| r_{j}^{n}-v_{j+1}^{-} r_{j+1}^{n}-v_{j-1}^{+} r_{j-1}^{n}\right], \\
\left.v_{i}\right|_{j} ^{n+1} & =S_{i}^{n}+\left.v_{i}\right|_{j+1} ^{n} \eta_{j}^{+}+\left.v_{i}\right|_{j-1} ^{n} \eta_{j}^{-}+\left.v_{i}\right|_{j} ^{n} \eta_{j}^{0},
\end{aligned}
$$

where $v_{j-1}^{+}=\max \left(\left.v_{1}\right|_{j-1} ^{n}, 0\right), v_{j+1}^{-}=-\min \left(\left.v_{1}\right|_{j+1} ^{n}, 0\right)$ and where $\eta_{j}^{\{+,-, 0\}}$ are random numbers defined by:

$$
\left(\eta_{j}^{+}, \eta_{j}^{-}, \eta_{j}^{0}\right)=\left\{\begin{array}{lll}
(1,0,0), & \text { with probability } P_{j}^{+} \\
(0,1,0), & \text { with probability } P_{j}^{-} \\
(0,0,1), & \text { with probability } P_{j}^{0}=1-P_{j}^{+}-P_{j}^{-}
\end{array} .\right.
$$


The probabilities $P_{j}^{0}, P_{j}^{-}$and $P_{j}^{+}$define the random choice method. They are given by:

$$
\begin{aligned}
P_{j}^{+} & =\frac{r_{j+1}^{n}}{r_{j}^{n+1}} v_{j+1}^{+} \frac{\Delta t}{\Delta x}, \\
P_{j}^{-} & =\frac{r_{j-1}^{n}}{r_{j}^{n+1}} v_{j-1}^{-} \frac{\Delta t}{\Delta x}, \\
P_{j}^{0} & =\frac{r_{j}^{n}}{r_{j}^{n+1}}\left(1-\left|\left(v_{1}\right)_{j}^{n}\right| \frac{\Delta t}{\Delta x}\right) .
\end{aligned}
$$

To guarantee that the probability $P_{j}^{0}$ is positive, one must enforce the following CFL condition:

$$
\left|\left(v_{1}\right)_{j}^{n}\right| \frac{\Delta t}{\Delta x} \leq 1
$$

Finally, the source term $S_{i}^{n}$ is defined as:

$$
S_{i}^{n}=-\frac{C_{1}}{2} \bar{\omega}^{n} v_{i}^{n} \Delta t+\sqrt{C_{0} \bar{\varepsilon}^{n} \Delta t} \xi_{i}^{n}-\left\langle\left. v_{i}\right|_{j+1} ^{n} \eta_{i}^{+}+\left.v_{i}\right|_{j-1} ^{n} \eta_{i}^{-}+\left.v_{i}\right|_{j} ^{n} \eta_{i}^{0}\right\rangle_{N_{s}}
$$

where $\xi_{i}^{n}$ are random independent Gaussian noises with variance 1 and mean 0 and where, for any quantity $q$, $\langle q\rangle_{N_{s}}$ represents the weighted mean on the stochastic field:

$$
\langle q\rangle_{N_{s}}=\frac{\sum_{s=1}^{N_{s}} r^{(s)} q^{(s)}}{\sum_{s=1}^{N_{s}} r^{(s)}} .
$$

The last term in equation (B.2) is a discretization of $\frac{\partial R_{1 i}}{\partial x_{1}}$ that guarantees that advection has a zero contribution to the evolution of the mean, as should be the case.

The numerical scheme described here can be shown to be first order in time and in space when stochastic convergence is achieved.

\section{Deterministic DiReCt Method}

We propose here a Finite Volume numerical method to discretize the equation (4.1), where the space, velocity fluctuation and time dimensions are discretized to yield a unique value of the $\operatorname{PDF} f_{1}\left(u_{1} ; x_{1}, t\right)$. This numerical scheme should allow to satisfy the following constraints:

$$
\begin{aligned}
f_{1}\left(u_{1} ; x_{1}, t\right) & \geq 0 \\
\int_{\mathbb{R}} f_{1}\left(u_{1} ; x_{1}, t\right) d u_{1} & =1, \\
\int_{\mathbb{R}} u_{1} f_{1}\left(u_{1} ; x_{1}, t\right) d u_{1} & =0 .
\end{aligned}
$$

To simplify notations, we will hereafter drop the index 1 from $x_{1}$ and $f_{1}$.

We introduce a Cartesian, uniform mesh, defined by the control volumes $\mathcal{C}_{i, j}=\left[x_{i-1 / 2}, x_{i+1 / 2}\right]\left[u_{j-1 / 2}, u_{j+1 / 2}\right]$, where $(i, j) \in I \times J \subset \mathbb{N} \times \mathbb{Z}$. We define $\Delta x$ and $\Delta v$ as the sizes of the space and velocity control volumes, respectively. $x_{i}=i \Delta x$ and $u_{j}=j \Delta v$ here refer to the cell centres, whereas $x_{i+1 / 2}=(i+1 / 2) \Delta x$ and $u_{j+1 / 2}=(j+1 / 2) \Delta u$ refer to the volume control boundaries. 
Let $f_{i, j}^{n}$ be an average approximation of the PDF on the control volume at time $t^{n}=n \Delta t, n \in \mathbb{N}$,

$$
f_{i, j}^{n}=\frac{1}{\Delta x \Delta v} \int_{\mathcal{C}_{i, j}} f\left(x, u, t^{n}\right) d u d x
$$

We start from the Finite Volume scheme originally derived in [17]. We recall the basic steps leading to its construction on the simplified advection equation

$$
\frac{\partial f}{\partial t}+u \frac{\partial f}{\partial x}=0, u>0
$$

for the sake of simplicity. Its extension by symmetry to the negative velocity space is straightforward. Its application to the right-hand side, velocity drift terms, in Equation (4.1), will be discussed hereafter.

First, a time explicit Euler scheme is employed to discretize the Equation (C.5) as

$$
f_{i}^{n+1}=f_{i}^{n}+u \frac{\Delta t}{\Delta x}\left(F_{i+1 / 2}^{n}-F_{i-1 / 2}^{n}\right)
$$

where $F_{i+1 / 2}^{n}=F\left(x_{i+1 / 2}, t^{n}\right)$ stands as a discrete conservative approximation of $f(x, t)$ on the boundary of the control volume $\left[x_{i-1 / 2}, x_{i+1 / 2}\right]$. Second, following [17], a second order MUSCL reconstruction technique (by primitive), leads to the approximation

$$
F\left(x, t^{n}\right)=\left[f_{i}^{n}+\epsilon^{+} \frac{x-x_{i}}{\Delta x}\left(f_{i+1}^{n}-f_{i}^{n}\right)\right], \forall x \in\left[x_{i-1 / 2}, x_{i+1 / 2}\right] .
$$

The slope limiter $\epsilon^{+}$is introduced in order to recover the maximum principle $0 \leq f_{i}^{n} \leq\|f\|_{\infty}$ under the CFL condition $u \frac{\Delta t}{\Delta x} \leq 1$. Its expression, given by

$$
\epsilon^{+}=\left\{\begin{array}{l}
0, \text { if }\left(f_{i+1}^{n}-f_{i}^{n}\right)\left(f_{i}^{n}-f_{i-1}^{n}\right)<0 \\
\min \left(1, \frac{2\left(\|f\|_{\infty}-f_{i}^{n}\right)}{f_{i}^{n}-f_{i+1}^{n}}\right), \text { if }\left(f_{i+1}^{n}-f_{i}^{n}\right)<0 \\
\min \left(1, \frac{2 f_{i}^{n}}{f_{i+1}^{n}-f_{i}^{n}}\right), \text { else }
\end{array}\right.
$$

leads to a non-linear expression for the numerical flux.

This approximation procedure can be further extended to evaluate the velocity drift term in the right hand side of Equation (4.1), which involves the velocity variance gradient $\frac{\partial \overline{u^{2}}}{\partial x}$. This drift term should balance with the advection term in the left hand side of Equation (4.1), in order to guarantee the zero mean velocity conservation (C.3). At the discrete level, this requirement is met with a re-definition of $\left.\frac{\partial \overline{u^{2}}}{\partial x}\right|_{i} ^{n}$ as a function of the discrete, reconstructed, numerical flux obtained for the advection term (left hand side of Equation (4.1))

$$
\left.\frac{\partial \overline{u^{2}}}{\partial x}\right|_{i} ^{n}=\sum_{j} u_{j}^{2} \frac{F_{i+1 / 2, j}^{n}-F_{i-1 / 2, j}^{n}}{\Delta x} \Delta v /\left(-\sum_{j} u_{j} \frac{F_{i, j+1 / 2}^{n}-F_{i, j-1 / 2}^{n}}{\Delta v} \Delta v\right)
$$

which is the analogous of the continuous relation, obtained by integration by parts,

$$
\frac{\partial \overline{u^{2}}}{\partial x}=\int_{\mathbb{R}} d u u^{2} \frac{\partial f}{\partial x} /\left(-\int_{\mathbb{R}} d u u \frac{\partial f}{\partial u}\right)
$$


The chosen discrete definition (C.9) mimics the integration by part (C.10) required to satisty the zero mean velocity conservation (C.3), that is $\bar{u}=0$.

A similar procedure is now applied to the $C_{1}$ term in the right hand side of Equation (4.1), which is rewritten as

$$
\frac{\partial}{\partial u}\left(\frac{C_{1}}{2} \bar{\omega} u f\right) \rightarrow \frac{\partial}{\partial u}\left(\frac{C_{1}}{2} \bar{\omega}(u-\bar{u}) f\right) .
$$

An extension of the Finite Volume scheme (C.6)-(C.7)-(C.8) is employed here, to the more general case where the fluxes depend on the drift variable $u$. This dependence is treated with a conservative centered discretization of the velocity variable in the flux uf. A discrete definition for $\bar{u}$ is required at this point in (C.11). We introduce an approximation that satisfies the zero mean velocity conservation in a discrete manner on the discrete analogous of the Equation (C.11)

$$
\bar{u}_{i}^{n}=\sum_{j} u_{j} \frac{F_{i, j+1 / 2}^{n}-F_{i, j-1 / 2}^{n}}{\Delta v} \Delta v /\left(\sum_{j} u_{j} \frac{u_{j+1 / 2} F_{i, j+1 / 2}^{n}-u_{j-1 / 2} F_{i, j-1 / 2}^{n}}{\Delta v} \Delta v\right)
$$

which is the discrete analogous of the continuous expression

$$
\bar{u}=\int_{\mathbb{R}} d u u \frac{\partial f}{\partial u} / \int_{\mathbb{R}} d u \frac{\partial}{\partial u}(u f)=0 .
$$

We finally obtain an unsplit discretization for all the advection terms in Equation (4.1). The discrete analogous of the probability density conservation (C.2) is satisfied if the slope limiters are not active for the advection term $u \frac{\partial f}{\partial x}$. In this case, we indeed obtain a centered discretization whatever the sign of the velocity is. We accept a small deviation from the probability density conservation, where the limiters are active to guarantee the maximum principle.

We now turn to the discretization of the $C_{0}$ operator in the right hand side of Equation (4.1). This term is splitted and discretized with an centered, implicit scheme, with net flux boundary conditions on the velocity space. This ensures the respect of the conservations (C.2) and (C.3), at the discrete level. Moreover, we obtain a M-matrix with a positive right hand side, leading to a positive PDF.

Finally, we remark that the splitting of the $C_{0}$ operator is convenient in the sense that it allows both the implicitation of this term and an easy implementation of a parallelisation on the space dimension $x$ with good expected scalability. We have made use of the MPI parallelisation protocol to do so.

Acknowledgements: This work is part of a CEMRACS project done while the authors were visiting the CIRM at Marseille. The authors acknowledge the support of CEA, DAM, DIF in financing the visit and the project.

This work was granted access to the HPC resources of Aix-Marseille Université financed by the project Equip@Meso (ANR-10-EQPX-29-01) of the program "Investissements d'Avenir" supervised by the Agence Nationale pour la Recherche.

\section{REFERENCES}

[1] S. B. Pope. PDF methods for turbulent reactive flows. Prog. Energ. Combust., 27:119-192, 1985.

[2] S.B. Pope. On the relationship between stochastic Lagrangian models of turbulence and second-moment closures. Phys. Fluids, 6:973-985, 1994.

[3] S. B. Pope. Turbulent flows. Cambridge Univ. Press, 2000.

[4] R. O. Fox and P. K. Yeung. Improved lagrangian mixing models for passive scalars in isotropic turbulence. Phys. Fluids, 15(4):961-985, 2003.

[5] B.A. Younis, T.B. Gatski, and C.G. Speziale. Towards a rational model for the triple velocity correlations of turbulence. Technical Report TM-1999-209134, NASA, April 1999. 
[6] I. Vallet. Reynolds stress modeling of three-dimensional secondary flows with emphasis on turbulent diffusion closure. J. App. Mech., 74:1142-1155, November 2007.

[7] P.R. Van Slooten, Jayesh, and S.B. Pope. Advances in PDF modeling for inhomogeneous turbulent flows. Phys. Fluids, 10(1):246-265, 1998.

[8] K. Hanjalic and B.E. Launder. A Reynolds stress model of turbulence and its application to thin shear flows. J. Fluid Mech., 52:609-638, 1972 .

[9] G.I. Barenblatt. Self-similar turbulence propagation from an instantaneous plane source,. Nonlinear Dynamics and Turbulence, pages 48-60, 1983.

[10] C. Cherfils and A.K. Harrison. Comparison of different statistical models of turbulence by similarity methods. In Proceedings of the Fluids Engineering Division Summer Meeting, Incline Village, Nevada, USA, June 1994. ASME.

[11] L. Valiño. A field Monte Carlo formulation for calculating the probability density function of a single scalar in a turbulent flow. Flow, turbulence and combustion, 60:157-172, 1998.

[12] V. A. Sabel'nikov and O. Soulard. Rapidly decorrelating velocity field model as a tool for solving Fokker-Planck PDF equations of turbulent reactive scalars. Phys. Rev. E, 72:016301, 2005.

[13] O. Soulard and V. A. Sabel'nikov. Eulerian Monte Carlo method for the joint velocity and mass-fraction probability density function in turbulent reactive gas flow combustion. Explosion and Shock Waves, 42(6):753-762, 2006.

[14] J.L. Lumley. Computational modeling of turbulent flows. Adv. Appl. Mech., 18:123-176, 1978.

[15] L. Cheng, Hailiang L., and S. Osher. Computational high-frequency wave propagation using the level set method, with applications to the semi-classical limit of Schrödinger equations. Comm. Math. Sci., pages 593-621, 2003.

[16] J. Glimm. Solutions in the large for nonlinear hyperbolic systems of equations. Comm. Pure Appl. Math., 18:697-715, 1965.

[17] F. Filbet, E. Sonnendrücker, and P. Bertrand. Conservative numerical schemes for the Vlasov equation. J. Comp. Phys., 172(1):166-187, 2001. 